\title{
Clinical Analysis and Surgical Considerations of Atherosclerotic Cerebral Aneurysms: Experience of a Single Center
}

\author{
Chang Kyu Park', Hee Sup Shin², Seok Keun Choi', Seung Hwan Lee ${ }^{2}$, Jun Seok Koh ${ }^{2}$ \\ 'Department of Neurosurgery, KyungHee University Hospital, Seoul, Korea \\ ${ }^{2}$ Department of Neurosurgery, KyungHee University Hospital at Gangdong, Seoul, Korea
}

Objective : Atherosclerotic cerebral aneurysms are known to increase occurrence of thromboembolic events and occlusion of perforator vessels intraoperatively due to pathological changes in the vessels themselves. In the current study, we analyzed the points to be considered during surgery for atherosclerotic cerebral aneurysms and the postoperative results.

Materials and Methods: We retrospectively reviewed the medical records, radiological results, and surgical records, including intraoperative video recordings and photographs, of 262 patients who underwent cerebral aneurysm surgery. We then performed a detailed analysis of aneurysm features, surgical methods, and clinical outcomes.

Results : Among 278 aneurysms in 262 patients, 73 aneurysms in 67 patients showed atherosclerotic features (atherosclerotic group, AG), and 205 aneurysms in 195 patients showed no evidence of atherosclerosis (non-atherosclerotic group, NAG). In the AG, clipping with multiple permanent clips was performed in 14 aneurysms, and clip slippage was found in four cases. Six AG cases had a remnant neck after clipping, which was significantly more frequent than in the NAG $(\rho<0.05)$. Clinical outcomes and surgery-related complications did not differ significantly between the two groups.

Conclusion : In the surgical repair of aneurysms, the incidence of ischemia, which is irreversible or severe, might be greater in atherosclerotic than in non-atherosclerotic aneurysms. In addition, multiple clips might be applied to atherosclerotic aneurysms for effective obliteration and an aneurysm neck might be left to avoid a region of atheroma.

Keywords Intracranial aneurysm, atherosclerosis, surgical procedure
J Cerebrovasc Endovasc Neurosurg. 2014 September; 16(3):247-253

Received : 3 July 2014

Revised : 29 August 2014

Accepted : 4 September 2014

Correspondence to Hee Sup Shin Department of Neurosurgery, Kyung Hee University Hospital at Gangdong, 149, Sangil -dong, Gangdong-gu, Seoul 134-727, Korea

Tel : 82-2-440-8402

Fax : 82-2-440-8404

E-mail : realeponym@hanmail.net

ORCID : http://orcid.org/0000-0002-5286-8448
This is an Open Access article distributed under the terms of the Creative Commons Attribution NonCommercial License (http://creativecommons.org/licenses/by-nc/3.0) which permits unrestricted noncommercial use, distribution, and reproduction in any medium, provided the original work is properly cited.

\section{INTRODUCTION}

The mortality rate following neurosurgical treatment of an unruptured cerebral aneurysm varies from $0.4 \%$ to $1.5 \%$, and age of the patient and size and location of the aneurysm are generally accepted factors that af- fect surgical outcome. ${ }^{9)}$ For achievement of acceptable surgery-related morbidity and mortality rates, neurosurgeons strive for precise aneurysmal neck clipping, careful confirmation of branches or perforators that were not clipped together, and maintenance of flow in the parent artery to prevent ischemic accidents. 

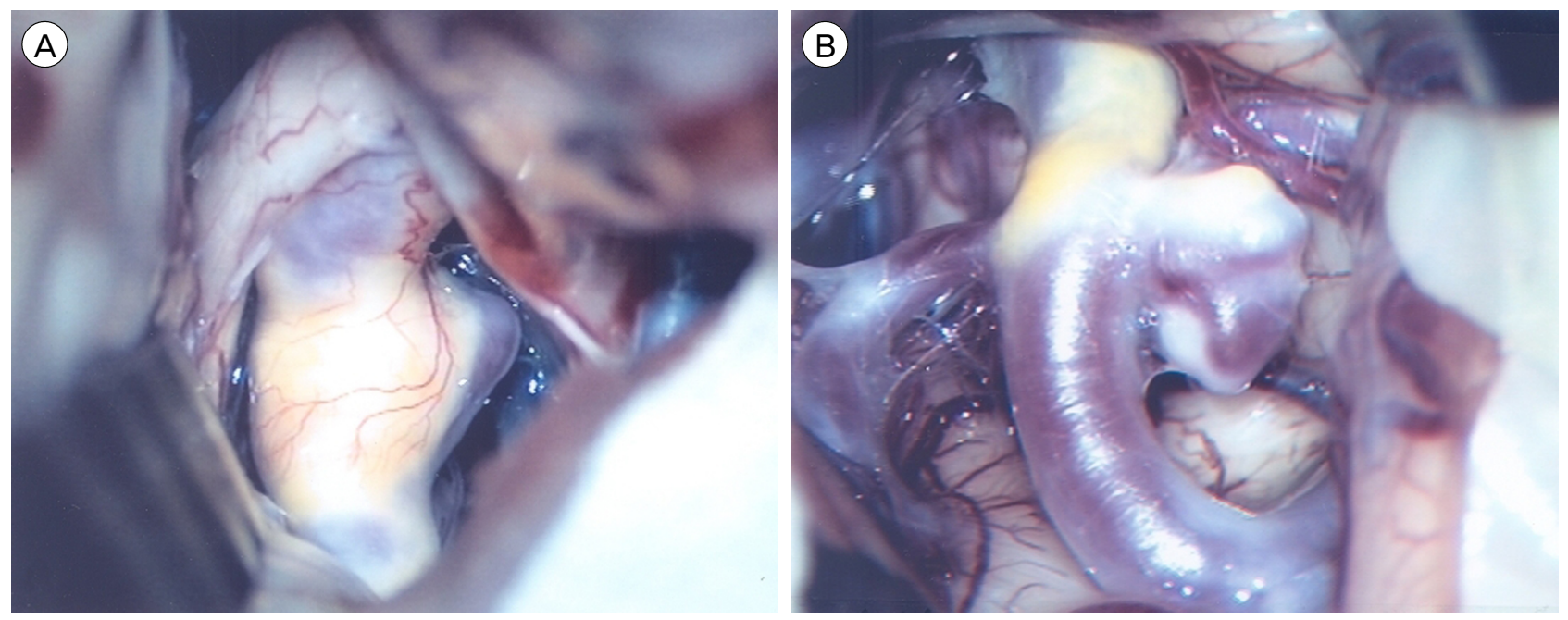

Fig. 1. Operative view of an aneurysm with atherosclerotic wall. (A) Atherosclerotic change in the parent artery and aneurysmal neck. (B) Atherosclerotic change in the parent artery and aneurysmal wall.

Atherosclerotic changes in the intracranial artery and in part of the aneurysm result in diminished flexibility of the vessel itself, which not only makes the surgery difficult to perform but can also result in unexpected ischemic complications such as thromboembolism and vessel occlusion. ${ }^{2)}$ In the current study, we analyzed and reported the clinical features of atherosclerotic intracerebral aneurysms treated at our center.

\section{MATERIALS AND METHODS}

\section{Subjects}

Cases of 262 patients who underwent aneurysmal neck clipping surgery at our center between June 2004 and November 2010 were analyzed retrospectively. We reviewed intraoperative videos; microscopic photographs; pre- and post-operative findings of computed tomography angiogram (CTA), magnetic resonance angiogram (MRA), and digital subtraction angiogram (DSA); and medical and surgical records. We divided the patients into two groups: the atherosclerotic group (AG) with atherosclerotic appearance of vessels, and the non-atherosclerotic group (NAG) without atherosclerosis.

\section{Evaluation of atherosclerotic aneurysm}

The criteria for atherosclerotic aneurysm were assessed according to the surgeons' intraoperative findings, either retrospectively or prospectively. Atherosclerotic changes in the vessel or aneurysm walls were identified as follows: (1) yellowish and/or whitish spotty appearance of the vessel and/or aneurysm; (2) surgical confirmation of a definite decrease in elasticity of the wall, with or without a color change of the vessel (Fig. 1).

Atherosclerosis types were divided according to the following categories based on the position of the atherosclerotic changes: (1) parent artery and aneurysm neck; (2) aneurysm neck; (3) aneurysm neck and dome; (4) aneurysm dome. Location in the parent artery was defined as atherosclerotic change within 5 $\mathrm{mm}$ from the neck of the aneurysm.

\section{Evaluation of surgical result}

Clinical outcome was determined according to scoring and changes on the Glasgow outcome scale (GOS). The outcome was determined as favorable if the GOS score was either unchanged, regardless of the preoperative status, or improved to a GOS score of 4-5. An outcome was considered unfavorable if there was deterioration to a GOS score of 1-3. In addition, the occurrence of new postoperative ischemia was assessed with a head computed tomography (CT) scan. The postoperative $\mathrm{CT}$ was analyzed for territory infarction or perforator infarction in relation to the clip position. 
Table 1. Demographic and aneurysm characteristics

\begin{tabular}{lccc}
\hline & AG $(\mathrm{n}=73)$ & NAG $(\mathrm{n}=205)$ & Sig. $(\boldsymbol{p}$ value) \\
\hline Age, yrs, mean $\pm \mathrm{SD}^{*}$ & $60.0 \pm 9.0$ & $54.2 \pm 11.3$ & 0.001 \\
Sex, $\mathrm{n}$, male/female $^{\dagger}$ & $27 / 40$ & $64 / 131$ & 0.299 \\
${\text { Ruptured aneurysm, } \mathrm{n}(\%)^{\dagger}}^{\dagger}$ & $48(65.8)$ & $143(69.8)$ & 0.558 \\
${\text { Location of aneurysm, } \mathrm{n}(\%)^{\ddagger}}_{\mathrm{MCA}}$ & & 0.012 \\
$\mathrm{ICA}$ & $43(58.9)$ & $93(45.4)$ & \\
$\mathrm{ACA}$ & $18(24.7)$ & $40(19.5)$ & \\
\hline
\end{tabular}

${ }^{*}$ Independent $t$ test used for statistical analysis, ${ }^{\dagger}$ Fisher's exact test used for statistical analysis, ${ }^{\ddagger}$ Pearson's Chi-square used for statistical analysis. $A G=$ atherosclerotic group; $N A G=$ non-atherosclerotic group; $M C A=$ middle cerebral artery; $I C A=$ internal carotid artery; $A C A=$ anterior cerebral artery; $y$ rs = years; $S D=$ standard deviation; Sig = significance; $\mathrm{n}=$ number

\section{Statistical analysis}

To evaluate the difference between the AG and NAG, the variables of clinical characteristics, aneurysmal characteristics, and surgical procedures between the two groups were compared using univariate methods (the two-sample $t$-test, Fisher's exact test, and Pearson's Chi-square test). MedCalc ${ }^{\circledR}$ (version 13, MedCalc Software bvba, Ostend, Belgium) was used for analysis, and a $p$ value less than 0.05 was considered statistically significant for all statistics.

\section{RESULTS}

\section{Patient characteristics}

Among 262 patients who underwent aneurysm clipping surgery at our center between June 2004 and November 2010, atherosclerotic aneurysms were found in 67 patients, representing approximately $25.6 \%$ of all patients undergoing aneurysm clipping surgery. The mean age of the patients with atherosclerotic aneurysms was $60.0 \pm 9.0$ years (mean $\pm S D$, range: 38-74), among whom 27 were males and 40 were females. The mean age of the remaining 195 patients without atherosclerotic aneurysms was $54.2 \pm$ 11.3 years (mean $\pm S D$, range: 21-79), among whom 64 were males and 131 were females (Table 1).

\section{Characteristics of aneurysms}

A total of 73 lesions were identified among the atherosclerotic aneurysms; of these, 48 lesions were rup- tured and the remaining 25 were unruptured. Among the non-atherosclerotic aneurysms, 205 lesions were identified; of these, 143 and 62 were ruptured and unruptured, respectively.

Among the atherosclerotic aneurysms, 43 were found in the middle cerebral artery (MCA), 18 were found in the internal carotid artery (ICA), and 12 were found in the anterior cerebral artery (ACA). Among the non-atherosclerotic aneurysms, 93 were found in the MCA, 40 were found in the ICA, and 72 were found in the ACA (Table 1). A significant difference in aneurysm location was observed between the two groups $(p=0.012)$.

\section{Characteristics of atherosclerotic changes}

When the vessels with atherosclerotic changes were visualized intraoperatively, sites of the changes varied considerably. Atherosclerotic changes were observed in the parent artery and aneurysm neck in 24 cases, in the aneurysm neck in 21 cases, in the aneurysm neck and dome in 17 cases, and in only the aneurysm dome in 11 cases (Table 2).

Table 2. Characteristics of atherosclerotic changes

\begin{tabular}{lc}
\hline \multicolumn{1}{c}{ Atherosclerotic change, $\mathrm{n}(\%)$} & Lesions $(\mathrm{n}=\mathbf{7 3})$ \\
\hline Parent artery and aneurysm neck & $24(32.9)$ \\
Aneurysm neck & $21(28.8)$ \\
Aneurysm neck and dome & $17(23.3)$ \\
Aneurysm dome & 11 (15.0) \\
\hline
\end{tabular}


Table 3. Characteristics of surgical steps*

\begin{tabular}{lccc}
\hline & AG $(\mathbf{n}=73)$ & NAG $(\mathbf{n}=\mathbf{2 0 5})$ & Sig. $(\boldsymbol{p}$ value) \\
\hline Multiple permanent clips and/or clip repositioning & $14(19.2)$ & $34(16.6)$ & 0.594 \\
Intentional remnant neck & $6(8.2)$ & $5(2.4)$ & 0.040 \\
Clip slippage & $4(5.5)$ & $4(1.9)$ & 0.213 \\
Wrapping & $1(1.3)$ & $8(3.9)$ & 0.453 \\
\hline
\end{tabular}

*Fisher's exact test used for statistical analysis.

AG = atherosclerotic group; NAG = non-atherosclerotic group; Sig = significance; $n=$ number

\section{Surgical procedure}

Out of 73 atherosclerotic aneurysms, 14 were clipped with multiple permanent clips; however, remnant neck and clip slippage were later observed in six and four cases, respectively. Among 205 surgeries for non-atherosclerotic aneurysms, multiple clippings were performed in 34 cases, remnant neck was found in five cases, and clip slippage occurred in four cases. Comparison of the two groups showed a statistically significant difference in the variable of intentional remnant neck (Table 3). We then subdivided the specific surgical steps in the atherosclerotic aneurysm group; there were 15 cases of specific surgical steps in the group with atherosclerotic change in the parent artery and aneurysmal neck. Among them, multiple clippings were performed in seven cases, remnant neck was found in four cases, and clip slippage occurred in three cases, with only one case of wrapping. In another group, atherosclerotic change on the aneurysmal neck, multiple clippings were performed in six cases, remnant neck was found in two cases. In the other group, atherosclerotic change on the aneurysmal neck and dome, multiple clippings were performed in one case and clip slippage occurred in one case (Table 4).

\section{Post-operative outcome}

In review of the postoperative outcomes of patients in the AG, 47 patients had GOS score of $4-5$ and 20 patients had GOS score of 1-3. One patient expired because of a territory infarction. In the AG, postoperative ischemic lesions developed in five patients; two of these patients had a total territory infarction (Fig. 2) and three patients had a perforator infarction related to the site of the operated lesion. In the NAG, five patients had infarctions in small subcortical regions. However, all patients in the NAG who developed postoperative infarctions recovered completely from their neurologic deficits. No statistical difference in development of ischemic complications was observed between the two groups.

\section{DISCUSSION}

Recently, treatment of cerebral aneurysms has been developing at a rapid pace, and a number of studies have reported good clinical outcomes of aneurysm surgeries. However, Flamm et al. reported that the presence of plaque in an aneurysm can be a pre-

Table 4. Characteristics of surgical steps according to atherosclerotic location*

\begin{tabular}{lcc}
\hline \multicolumn{1}{c}{ Atherosclerotic change } & Specific surgical steps ${ }^{\dagger}, \mathbf{n}(\%)$ & Usual surgical steps, $\mathbf{n}(\%)$ \\
\hline Parent artery and aneurysm neck & $15(63 \%)$ & $9(37 \%)$ \\
Aneurysm neck & $8(38 \%)$ & $13(62 \%)$ \\
Aneurysm neck and dome & $2(12 \%)$ & $15(88 \%)$ \\
Aneurysm dome & 0 & $11(100 \%)$ \\
\hline
\end{tabular}

*Pearson's Chi-square test used for statistical analysis. Statistical difference was revealed among the groups with $\rho$ value of 0.0004 .

${ }^{\dagger}$ The specific surgical steps included 1) multiple permanent clips and/or clip repositioning, 2) intentional remnant neck, 3) clip slippage, and 4) wrapping.

$\mathrm{n}=$ number 


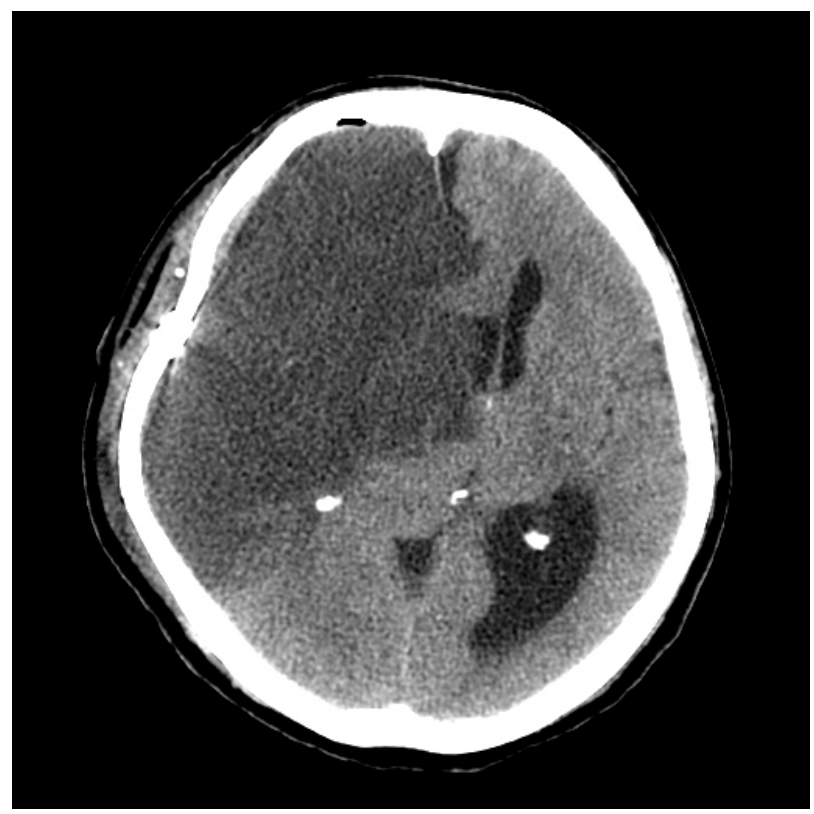

Fig. 2. Postoperative head computed tomography (CT) scan of a patient who underwent surgical neck clipping for a right anterior choroidal artery aneurysm which showed atherosclerotic change in the parent artery and aneurysmal neck. He then developed neurologic deficits postoperatively. It shows a low-density lesion of the right internal carotid artery territory. Right cerebral swelling creates a mass effect, resulting in midline shifting and ipsilateral ventricle compression.

dictive factor for unexpected complications during aneurysm surgery. ${ }^{3)}$ Other researchers have also examined the importance of atherosclerotic aneurysms.

In 1999, Ohno et al. reported atherosclerotic changes in six patients $(10 \%)$ among 30 patients with cerebral aneurysms. $^{8)}$ Similarly, in 2003, Grigorian et al. reported atherosclerotic changes in 81 patients $(26.7 \%)$ among 333 patients with aneurysms. ${ }^{4)}$ In our study, $67(25.6 \%)$ of the 262 patients with aneurysms had atherosclerotic aneurysms. Therefore, when treating aneurysms, understanding the pathophysiology of atherosclerotic changes is important.

Atherosclerosis is a well-known pathological condition that generally affects the arterial blood vessels. As a degenerative process, atherosclerotic changes result from deposition of plasma lipids, connective tissues, and local or circulating cells over the intima of the blood vessels, which leads to narrowing of the vessel lumen.7) The restorability of such atherosclerotic vessels is limited because of a resultant de- crease in elasticity and increase in rigidity of the vessel. ${ }^{10)}$ During aneurysm surgery, if the aneurysmal neck has atherosclerotic changes, problems such as incomplete neck clipping and narrowing of the lumen of the parent artery may occur. ${ }^{8)}$ In particular, narrowing of the lumen of the parent artery may be an important cause of postoperative ischemic events.

Regarding aneurysm location, Szelenyi et al. reported that among patients with atherosclerotic aneurysms, ten had aneurysms in the ICA, five had aneurysms in the anterior communicating artery, and 19 had aneurysms in the MCA.9) Ohno et al. reported that six patients among seven patients with atherosclerotic changes in the aneurysmal neck had MCA aneurysms. ${ }^{8}$ In our study, 43 patients among the 73 patients with atherosclerotic aneurysms had aneurysms in the MCA.

Szelenyi et al. reported that eight cases among 34 atherosclerotic aneurysm cases had atherosclerotic changes in the parent vessel, aneurysm dome, or aneurysm neck, respectively, and 11 cases had changes in both the aneurysm dome and neck. ${ }^{9)}$ In our study of 73 cases, the sites of atherosclerotic change were as follows: both parent artery and aneurysm neck in $24(32.9 \%)$, aneurysm neck in $21(28.8 \%)$, both aneurysm neck and dome in 17 (23.3\%), and aneurysm dome in $11(15.0 \%)$. These findings are not relevant to the findings reported in the papers mentioned earlier.

Regarding the postoperative prognosis of patients with atherosclerotic aneurysms, Szelenyi et al. reported that 27 of 34 atherosclerotic aneurysm patients had a good clinical outcome, increasing to 30 patients at six months. Postoperatively, four patients had an unfavorable outcome, and at the six-month follow-up, only three patients had an unfavorable outcome." In our series, 47 patients had a favorable outcome, 20 patients had an unfavorable outcome, and, unfortunately, one patient expired. The most likely explanation for the higher rate of unfavorable clinical outcomes in our series as compared with that of Szelenyi et al. is that the latter study included only 
unruptured aneurysms, whereas we included 48 cases of ruptured aneurysms. Reviews of other studies have found that the frequency of morbidity in patients with atherosclerotic aneurysms is higher than in patients with non-atherosclerotic aneurysms, approximately $14 \%$ and $6.8 \%$, respectively. ${ }^{23) 6}$

In atherosclerotic aneurysms, the atherosclerotic wall might increase the occurrence of thromboembolic events, or its rigidity might lead to occlusion of perfo- rators within the aneurysm vicinity during surgery. ${ }^{15)}$ Szelenyi et al. reported that in six patients with atherosclerotic aneurysms, a small residuum of the aneurysm neck was intentionally left. This was done significantly more often than in patients with non-atherosclerotic aneurysms. In addition, in patients with atherosclerotic aneurysms, temporary vessel occlusion and multiple repositioning of the permanent clip were performed more often than in patients with non-athe-
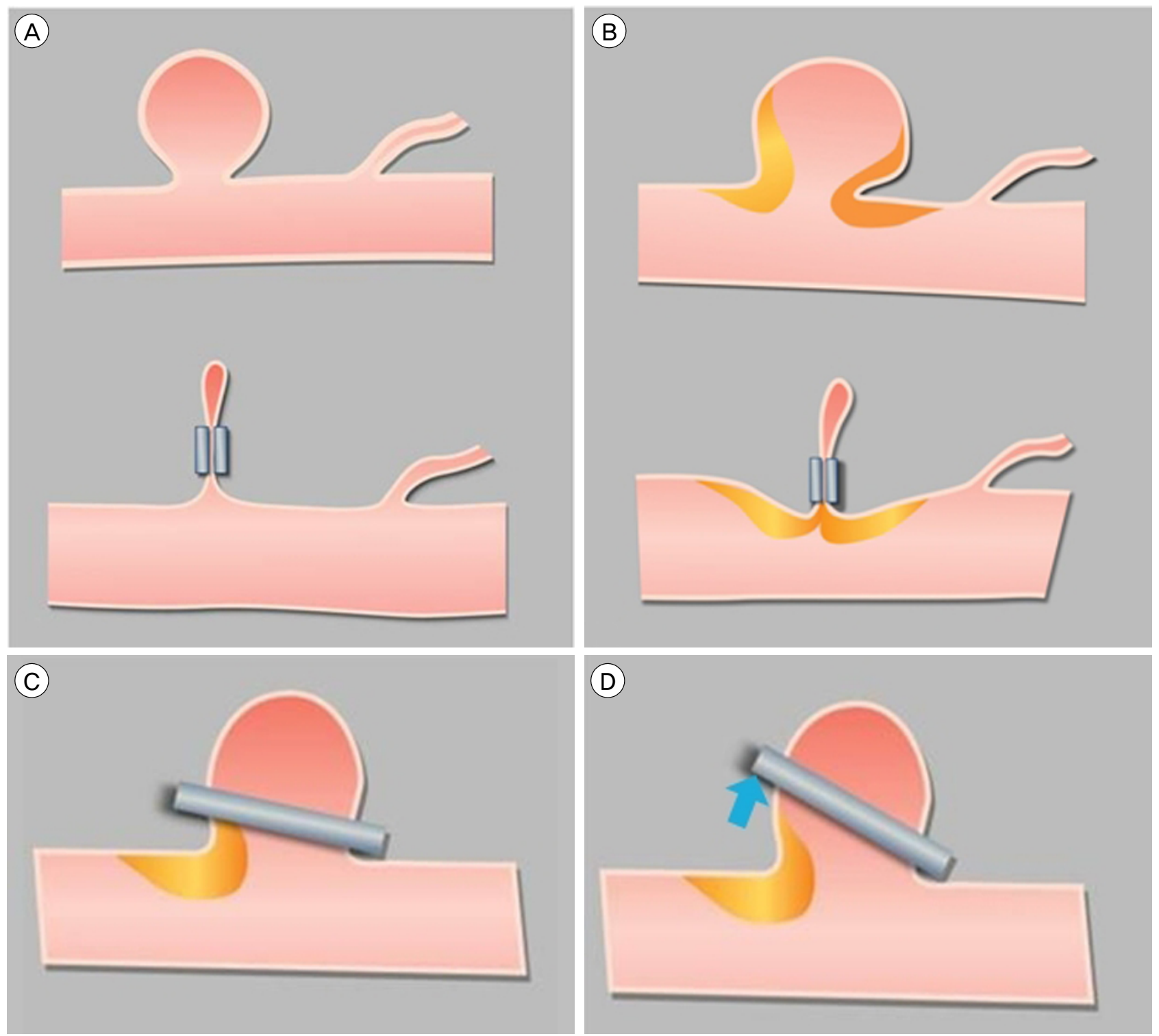

Fig. 3. Schematic illustrations of cerebral aneurysms. (A) Non-atherosclerotic aneurysm prior to neck clip placement (above) and with neck clip in place (below). (B) Parent artery narrowing due to atheroma, with and without neck clip in place. (C) and (D) Atherosclerotic change in the aneurysm neck leads to sliding of the clip and results in a remnant neck after clipping. 
rosclerotic aneurysms. Although the application of more than one permanent clip and wrapping occurred more often in patients with atherosclerotic aneurysms, this did not reach statistical significance in their study. ${ }^{9)}$ It is expected that clip placement in atherosclerotic aneurysms requires more surgical steps compared with non-atherosclerotic aneurysms. This is supported by our finding that the intraoperative observation of atherosclerosis showed significant correlation with a higher percentage of multiple positioning of a permanent clip. Clip placement might be hampered by a rigid atherosclerotic wall such that aneurysm obliteration due to sliding of the clip off the aneurysm dome is not complete or that the parent vessel or perforating branches are occluded (Fig. 3). One solution to this problem is aneurysm obliteration with multiple clips. ${ }^{89)}$ Furthermore, as in our study, avoidance of the region of the atheroma could promote effective clip placement and prevent parent artery occlusion.

\section{CONCLUSION}

In the surgical repair of aneurysms, the incidence of ischemia, which is irreversible or severe, might be greater in atherosclerotic than in non-atherosclerotic aneurysms. Careful manipulation and a thorough understanding of atherosclerotic vessels during neck clipping surgery can prevent ischemic accidents resulting from parent artery stenosis or perforating artery occlusion and yield satisfactory surgical results. In addition, multiple clips might be applied to atherosclerotic aneurysms for effective obliteration and the aneurysm neck might be left to avoid a region of atheroma.

\section{REFERENCES}

1. Bendszus M, Stoll G. Silent cerebral ischaemia: hidden fingerprints of invasive medical procedures. Lancet Neurol. 2006 Apr;5(4):364-72.

2. Carvi y Nievas MN, Hollerhage HG. Risk of intraoperative aneurysm clip slippage: a new experience with titanium clips. J Neurosurg. 2000 Mar;92(3):478-80.

3. Flamm ES, Grigorian AA, Marcovici A. Multifactorial analysis of surgical outcome in patients with unruptured middle cerebral artery aneurysms. Ann Surg. 2000 Oct;232(4) :570-5.

4. Grigorian AA, Marcovici A, Flamm ES. Intraoperative factors associated with surgical outcome in patients with unruptured cerebral aneurysms: the experience of a single surgeon. J Neurosurg. 2003 Sep;99(3):452-7.

5. Hadeishi H, Yasui N, Suzuki A. [Risks of surgical treatment for unruptured intracranial aneurysms]. No Shinkei Geka. 1991 Oct;19(10):945-9. Japanese.

6. King JT Jr, Berlin JA, Flamm ES. Morbidity and mortality from elective surgery for asymptomatic, unruptured, intracranial aneurysms: a meta-analysis. J Neurosurg. 1994 Dec;81(6):837-42.

7. Lindholt JS. Aneurysmal wall calcification predicts natural history of small abdominal aortic aneurysms. Atherosclerosis. 2008 Apr;197(2):673-8.

8. Ohno K, Arai T, Isotani E, Nariai T, Hirakawa K. Ischaemic complication following obliteration of unruptured cerebral aneurysms with atherosclerotic or calcified neck. Acta Neurochir (Wien). 1999 Jul;141(7):699-705; discussion 705-6.

9. Szelenyi A, Beck J, Strametz R, Blasel S, Oszvald A, Raabe $A$, et al. Is the surgical repair of unruptured atherosclerotic aneurysms at a higher risk of intraoperative ischemia? Clin Neurol Neurosurg. 2011 Feb;113(2):129-35.

10. Tateshima S, Tanishita K, Omura H, Sayre J, Villablanca $\mathrm{JP}$, Martin $\mathrm{N}$, et al. Intra-aneurysmal hemodynamics in a large middle cerebral artery aneurysm with wall atherosclerosis. Surg Neurol. 2008 Nov;70(5):454-62; discussion 462 . 